\title{
Three-dimensional direct numerical simulation study of conditioned moments associated with front propagation in turbulent flows
}

\author{
R. Yu, ${ }^{1, a)}$ A. N. Lipatnikov, ${ }^{2}$ and X. S. Bai ${ }^{1}$ \\ ${ }^{1}$ Division of Fluid Mechanics, Lund University, Lund, Sweden \\ ${ }^{2}$ Department of Applied Mechanics, Chalmers University of Technology, Goteborg, Sweden
}

(Received 11 May 2014; accepted 17 July 2014; published online 6 August 2014)

In order to gain further insight into (i) the use of conditioned quantities for characterizing turbulence within a premixed flame brush and (ii) the influence of front propagation on turbulent scalar transport, a 3D Direct Numerical Simulation (DNS) study of an infinitely thin front that self-propagates in statistically stationary, homogeneous, isotropic, forced turbulence was performed by numerically integrating Navier-Stokes and level set equations. While this study was motivated by issues relevant to premixed combustion, the density was assumed to be constant in order (i) to avoid the influence of the front on the flow and, therefore, to know the true turbulence characteristics as reference quantities for assessment of conditioned moments and (ii) to separate the influence of front propagation on turbulent transport from the influence of pressure gradient induced by heat release. Numerical simulations were performed for two turbulence Reynolds numbers (50 and 100) and four ratios (1,2, 5, and 10) of the rms turbulent velocity to the front speed. Obtained results show that, first, the mean front thickness is decreased when a ratio of the rms turbulent velocity to the front speed is decreased. Second, although the gradient diffusion closure yields the right direction of turbulent scalar flux obtained in the DNS, the diffusion coefficient $D_{t}$ determined using the DNS data depends on the mean progress variable. Moreover, $D_{t}$ is decreased when the front speed is increased, thus, indicating that the front propagation affects turbulent scalar transport even in a constant-density case. Third, conditioned moments of the velocity field differ from counterpart mean moments, thus, disputing the use of conditioned velocity moments for characterizing turbulence when modeling premixed turbulent combustion. Fourth, computed conditioned enstrophies are close to the mean enstrophy in all studied cases, thus, suggesting the use of conditioned enstrophy for characterizing turbulence within a premixed flame brush. (c) 2014 AIP Publishing LLC. [http://dx.doi.org/10.1063/1.4891735]

\section{INTRODUCTION}

In spite of the rapid development of Direct Numerical Simulation (DNS) approach to modeling premixed turbulent combustion, less expensive Reynolds-Averaged Navier-Stokes (RANS) approach $^{1,2}$ and Large Eddy Simulation (LES) ${ }^{3,4}$ are still major CFD tools at least in applied research. Such simulations require models for mean or filtered mass rate of product creation $\bar{W}$ or global or subgrid turbulent flame speed $S_{\mathrm{T}}$. Both $\bar{W}$ and $S_{\mathrm{T}}$ depend substantially on the rms velocity $U^{\prime}$, which is often considered to be the primary characteristic of turbulence in the combustion literature. Therefore, in order for a RANS or LES study of a premixed turbulent flame to be predictive, variations in this turbulence characteristic within the flame brush should be modeled properly.

However, as stressed elsewhere, ${ }^{5,6}$ a consistent definition of $U^{\prime}$ in a premixed turbulent flame is an issue, which can be understood by considering the case of fresh reactants and burned products

a) Author to whom correspondence should be addressed. Electronic mail: Rixin.yu@energy.lth.se. Fax: +46462224717. 
separated by an infinitely thin self-propagating interface (flamelet). As shown by Bray et al., ${ }^{7}$ application of definition of the rms turbulent velocity, which is standard for constant-density flows, i.e., $U^{\prime 2}(\mathbf{x})=\overline{\rho u_{k}^{\prime \prime} u_{k}^{\prime \prime}}(\mathbf{x}) / 3 \bar{\rho}(\mathbf{x})$, to the considered flame brush yields a quantity

$$
\begin{aligned}
& \frac{\overline{\rho u_{k}^{\prime \prime} u_{k}^{\prime \prime}}}{\bar{\rho}}=(1-\tilde{c})\left(\overline{u_{k}^{\prime} u_{k}^{\prime}}\right)_{u}+\tilde{c}\left(\overline{u_{k}^{\prime} u_{k}^{\prime}}\right)_{b}+\tilde{c}(1-\tilde{c})\left(\bar{u}_{k, b}-\bar{u}_{k, u}\right)\left(\bar{u}_{k, b}-\bar{u}_{k, u}\right) \\
& =(1-\tilde{c})\left(\overline{u_{k}^{\prime} u_{k}^{\prime}}\right)_{u}+\tilde{c}\left(\overline{u_{k}^{\prime} u_{k}^{\prime}}\right)_{b}+\frac{\overline{\rho u_{k}^{\prime \prime} c^{\prime \prime}} \cdot \overline{\rho u_{k}^{\prime \prime} c^{\prime \prime}}}{\bar{\rho}^{2} \tilde{c}(1-\tilde{c})}
\end{aligned}
$$

that is straightforwardly affected by the magnitude of slip velocity vector $\Delta \mathbf{u}=\overline{\mathbf{u}}_{b}-\overline{\mathbf{u}}_{u}$ and, therefore, depends not only on turbulence, but also on velocity jump across the flamelet. Here, $\rho$ is the density, $c$ is the combustion progress variable, $\mathbf{u}$ is the velocity vector, summation convention applies for the repeated index $k ; \bar{q}$ and $\tilde{q} \equiv \overline{\rho q} / \bar{\rho}$ designate the Reynolds and Favre average, respectively, of any variable $q$ with $q^{\prime \prime} \equiv q-\tilde{q}$, subscripts $u$ and $b$ designate quantities conditioned to unburned and burned states, respectively, $\left(\overline{u_{k}^{\prime} u_{k}^{\prime}}\right)_{u}$ and $\left(\overline{u_{k}^{\prime} u_{k}^{\prime}}\right)_{b}$ are the traces of the conditioned Reynolds-stress tensors $\left(\overline{u_{i}^{\prime} u_{j}^{\prime}}\right)_{u}=\left(\overline{u_{i} u_{j}}\right)_{u}-\bar{u}_{i, u} \bar{u}_{j, u}$ and $\left(\overline{u_{i}^{\prime} u_{j}^{\prime}}\right)_{b}=\left(\overline{u_{i} u_{j}}\right)_{b}-\bar{u}_{i, b} \bar{u}_{j, b}$, respectively.

Alternatively, conditioned second moment $\left(\overline{u_{k}^{\prime} u_{k}^{\prime}}\right)_{u}$ or $\left(\overline{u_{k}^{\prime} u_{k}^{\prime}}\right)_{b}$ is often considered to be the true turbulence characteristic in a flame and various experimental investigations, reviewed in Ref. 5, showed significant differences between the mean $\overline{\rho u_{k}^{\prime \prime} u_{k}^{\prime \prime}} / \bar{\rho}$ and conditioned, $\left(\overline{u_{k}^{\prime} u_{k}^{\prime}}\right)_{u}$ or $\left(\overline{u_{k}^{\prime} u_{k}^{\prime}}\right)_{b}$, turbulent kinetic energy. However, by considering very simple model problems, it was recently stressed that substantial difference still exists between conditioned and the true rms turbulent velocities. ${ }^{6}$ For instance, application of Eq. (1) to the case of equal densities of products and fresh mixture shows that $\left(\overline{u_{k}^{\prime} u_{k}^{\prime}}\right)_{u}$ or $\left(\overline{u_{k}^{\prime} u_{k}^{\prime}}\right)_{b}$ should differ from $\overline{u_{k}^{\prime} u_{k}^{\prime}}$ owing to the positive last term on the right-hand side (RHS); but $\overline{u_{k}^{\prime} u_{k}^{\prime}}$ is the true turbulence characteristic in this case, because flamelets do not affect velocity field when density is constant. If, moreover, the gradient diffusion closure of the turbulent scalar flux is invoked, which is typical for constant-density turbulent flows, then, Eq. (1) reads

$$
\overline{\frac{\rho u_{k}^{\prime \prime} u_{k}^{\prime \prime}}{\bar{\rho}}}=(1-\tilde{c})\left(\overline{u_{k}^{\prime} u_{k}^{\prime}}\right)_{u}+\tilde{c}\left(\overline{u_{k}^{\prime} u_{k}^{\prime}}\right)_{b}+\frac{D_{t}^{2}}{\tilde{c}(1-\tilde{c})}\left(\frac{\partial \tilde{c}}{\partial x_{k}}\right)^{2}
$$

thus, clearly showing that conditioned moments $\left(\overline{u_{k}^{\prime} u_{k}^{\prime}}\right)_{u}$ and $\left(\overline{u_{k}^{\prime} u_{k}^{\prime}}\right)_{b}$ are spatially non-uniform and depend on the mean combustion progress variable if the turbulent field $\overline{u_{k}^{\prime} u_{k}^{\prime}}$ is spatially uniform.

The substantial difference between conditioned and canonical mean turbulence characteristics results from the fact that conditional averaging is performed over spatial domain which boundary is wrinkled, randomly moves, with this motion being anisotropic. Due to the motion of the boundary, first, conditional averaging commutes neither with time nor with spatial derivatives, e.g., divergence of conditioned velocity vector does not vanish in a constant density flow in a general case. ${ }^{8}$

Second, due to the flux of a fluid through the boundary, e.g., conversion or reactants to products in flamelets, conditioned balance equations involve source or sink terms, which do not appear in the counterpart Reynolds-averaged equations. This was clearly shown in the pioneering papers by Libby $^{8}$ and Dopazo ${ }^{9}$ who introduced conditioned balance equations into fluid mechanics in order to address external intermittency in constant-density turbulent flows. As far as the propagation of a flamelet of a finite thickness in turbulent flow is concerned, proper conditioned balance equations were straightforwardly derived elsewhere ${ }^{10}$ see also Eqs. (215)-(220) in a recent review paper. ${ }^{5}$ One can easily show that these equations involve source and sink terms that do not vanish even in the constant-density case, see Eqs. (2)-(4), (12), and (13) in Ref. 11. These terms substantially change conditioned quantities when compared to the counterpart canonical Reynolds-averaged quantities. Even if turbulence is homogeneous and isotropic, the aforementioned source and sink terms are neither isotropic nor spatially uniform in the simplest statistically stationary $1 \mathrm{D}$ case. ${ }^{11}$

Target-directed research into the differences between the mean and conditioned rms velocities that (differences) are solely controlled by the difference between the conventional and conditional 
averaging techniques has yet been scarce in the combustion literature. Such differences were recently illustrated in Ref. 11 by applying conditioned balance equations ${ }^{10}$ to numerical simulations of a statistically planar, 1D, premixed turbulent constant-density "flame." Nevertheless, results obtained in Refs. 6 and 10 cannot be considered to be a decisive proof owing to the simplicity of the problem addressed in Ref. 6 and a number of closure assumptions invoked in Ref. 10.

Experimental research into the discussed problem requires the knowledge of the true turbulence characteristics within a premixed flame brush, but such characteristics have not yet been defined in a consistent manner in a general case. Such characteristics are confidently known only in a hypothetical constant-density case, which is difficult to study in an experiment. Therefore, DNS of front propagation in a constant-density turbulent flow appears to be a proper tool for gaining further insight into relation between conditioned and true turbulence characteristics.

Based on the above reasoning, a DNS study of a self-propagating front was recently carried out in 2D statistically planar constant-density turbulent flow. ${ }^{12}$ Substantial difference between conditioned and unconditioned moments of velocity field was confirmed for first, second, and third moments, with the difference depending on the front speed $S_{L}$ and turbulent Reynolds number Re. However, because inherently $3 \mathrm{D}$ phenomena such as vortex stretching play a substantial role in turbulence, the energy cascading can be fundamentally different in 2D and 3D cases, ${ }^{13}$ thus, putting the validity of 2D DNS results into question. Moreover, the previous 2D DNS study ${ }^{12}$ dealt with an open, inletoutlet configuration, with turbulence being fed through the inlet and decaying along the mean flow direction. In this case, the front movement is inherently unstable. ${ }^{14}$ To ankle the mean front position for sampling statistics, the mean inlet speed must be modulated using heuristic control strategy. ${ }^{14}$ Such oscillating velocity modulations mimic turbulence, thus, impeding interpretation of computed results.

To overcome the above difficulties and to gain further insight into the relation between conditioned and the true turbulence characteristics, we performed a DNS study of an infinitely thin self-propagating interface embedded into statistically homogenous, isotropic 3D turbulence. In order to know the true turbulence characteristics as reference ones, the assumption of a constant density was invoked.

Moreover, this assumption also offers an opportunity to look into effects of front propagation on turbulent scalar flux, by separating this phenomenon from the influence of heat release on the flux in a flame. Accordingly, another goal of the present work is to study the former effects.

Furthermore, while the influence of heat release on turbulent flow plays a substantial role in premixed combustion, as reviewed elsewhere, ${ }^{5}$ a study of the propagation of a front in a constantdensity turbulence is also of interest for assessing various models of premixed turbulent combustion that are widely used today. The point is that the vast majority of such models ${ }^{1,2}$ were developed by placing the focus of consideration on the influence of turbulence on burning, but ignoring the influence of heat release on turbulence. Therefore, the present constant-density DNS is a proper case for target-directed testing such models under conditions they address. This goal is mainly beyond the scope of the present work and will be addressed in subsequent publications, but one result relevant to modeling mean flame brush thickness will be reported in Sec. IV A.

Finally, the constant-density simplification allowed us to use fully periodic boundary conditions, thus, avoiding problems of front stabilization. Indeed, front elements that reach a boundary of the computational domain can simultaneously enter the domain through the opposite boundary owing to the lack of any influence of a constant-density front on the flow. Therefore, we consider such a 3D DNS setup to be an important contribution to numerical combustion.

\section{GOVERNING EQUATIONS}

Constant-density turbulent flow is governed by Navier-Stokes equations,

$$
\begin{gathered}
\nabla \cdot \mathbf{u}=0 \\
\partial_{t} \mathbf{u}+\mathbf{u} \cdot \nabla \mathbf{u}=-\frac{1}{\rho} \nabla p+v \nabla^{2} \mathbf{u}+\mathbf{f},
\end{gathered}
$$


where $v$ and $p$ are the kinematic viscosity and pressure, respectively, and term $\mathbf{f}(\mathbf{x}, \mathrm{t})$ is added in order to maintain constant turbulence intensity by using energy forcing at low wave numbers. Under such forcing, the injected energy cascades down to balance the dissipation at small scales, with the turbulence statistics at smaller scales being hardly affected. ${ }^{15}$ The forcing scheme is further discussed in Sec. III.

As conditioned moments are best defined in the case of an infinitely thin front, it is this problem that is simulated by numerically solving the level-set equation, ${ }^{16}$

$$
\partial_{t} G+\mathbf{u} \cdot \nabla G=S_{L}|\nabla G|,
$$

where $G$ is a signed distance function to the closest flame front $G(\mathbf{x}, t)=0$. The combustion progress variable $c(\mathbf{x}, t)=H[G(\mathbf{x}, t)-0]$ is defined using the Heaviside function $H(z)$. The front speed $S_{L}$ is kept constant. As far as relevance of such an assumption to premixed combustion is concerned, the case of $S_{L}=$ const. is associated with a laminar flame characterized by a zero Markstein number Ma with respect to unburned gas, e.g., near stoichiometric methane-air flame. It is worth also noting that theories of weakly perturbed laminar flames predict that variously defined Markstein numbers depend on the density ratio and Lewis number Le, with Ma vanishing in the constant-density equidiffusive (Le $=1$ ) case. ${ }^{17}$ Furthermore, because these theories yield $S_{L}=$ const. for infinitely thin flames, the present study of an infinitely thin front that propagates at a constant speed is consistent with these theories.

\section{NUMERICAL METHOD AND RUNNING CONDITIONS}

Simulations were performed using a simplified in-house DNS solver ${ }^{18}$ originally developed for low Mach number reacting flows with detailed chemistry and already applied to various reacting flow systems. ${ }^{19-22}$ The temporal integration of the governing equations is based on a second order symmetrical Strang splitting algorithm, originally designed to enable a separated chemistry solver. With one global time step, the integration of viscous terms can be automatically split into multiple sub-time steps of explicit integrations, with substep size given by the diffusion stability limit. Spatial discretization and interpolations are based on sixth order center schemes. The Poisson equation for pressure is solved with an efficient multigrid method. ${ }^{23}$ The DNS code is implemented in a vector form enabling $1 \mathrm{D}, 2 \mathrm{D}$, and 3D simulations. ${ }^{18}$

The computational domain is a square box of size $L_{\mathrm{x}} \times L_{\mathrm{y}} \times L_{\mathrm{z}}$ with $L_{\mathrm{x}}=4 L_{\mathrm{y}}=4$ $L_{\mathrm{z}}=4 L$, discretized on a uniform grid of $512 \times 128 \times 128$ cells. The initial turbulence field is generated by synthesizing Fourier waves ${ }^{24}$ with an initial rms velocity $U_{0}^{\prime}$ and integral length scale $\lambda_{0}=L / 4$. Following Ref. 25, the forcing function $\mathbf{f}(\mathbf{x}, t)=\sum_{\kappa} \hat{\mathbf{f}}_{\kappa}(t) e^{i \kappa \cdot \mathbf{x}}$ with

$$
\hat{\mathbf{f}}_{\kappa}(t)=\langle\varepsilon\rangle \hat{\mathbf{u}}_{\kappa}(t) 1_{\kappa-\kappa_{r}(t)} /\left(\hat{\mathbf{u}}_{\kappa}(t) \hat{\mathbf{u}}_{\kappa}^{*}(\mathrm{t})\right),
$$

is invoked in order to maintain statically stationary turbulence. Here, $\hat{\mathbf{f}}_{\kappa}$ is the Fourier mode of $\mathbf{f}$ in the wave number $\boldsymbol{\kappa}$-space, $\varepsilon$ is the dissipation rate, the bracket $\langle\cdot\rangle$ designates averaging over entire domain. The caret operator designates the complex Fourier mode $\hat{q}_{\kappa}(t)=\left\langle q(\mathbf{x}, t) e^{-i \kappa \cdot \mathbf{x}}\right\rangle$ for any $q$, $1_{\boldsymbol{\kappa}-\boldsymbol{\kappa}_{r}}=1$ when $\boldsymbol{\kappa}=\boldsymbol{\kappa}_{r}$, otherwise 0 , and $\boldsymbol{\kappa}_{r}$ is a randomly selected (at each time step) non-zero wave number vector within a lower wave number band $\left|\kappa_{r}\right| \leq \kappa_{f}=3 k_{0}=6 \pi / L$ and also in a form of $\boldsymbol{\kappa}_{r}=m_{i} k_{0}$ with $m_{i}$ as integer. As shown in Sec. IV, the forced turbulence reaches good statistical homogeneity/isotropy.

The $G$ field is initialized to give a planar flame surface at $x=L_{\mathrm{x}} / 2$ and $t=0$. Subsequently, the level-set equation (4) is solved within a narrow band of cells (six cells wide) covering the flame front ${ }^{26}$ followed by re-initialization $|\nabla G|=1$ of the neighboring $G$ field to a signed distance function. A third order weighted essential non-oscillating (WENO) scheme $^{27}$ and a third order total variation diminishing (TVD) type Runge-Kutta scheme ${ }^{28}$ are used for the spatial and temporal discretization of the $G$ equation, respectively. To guaranty that the zero level set coincides with the original one during signed-distance re-initialization, a sub-cell fix method ${ }^{29,30}$ is used.

To enable periodic propagation of the front through the computational domain in $x$ direction, (i) the progress variable is set using $x$-periodicity, i.e., $c(x, y, z, t)=c\left(x+m L_{x}, y, z, t\right)$ for any integer 
$m$ and $x \notin\left(0, L_{x}\right]$, and (ii) an instantaneous mean flame position $\xi$ is defined as follows

$$
\xi^{n+1}=\xi^{n}+\frac{3}{8} L_{x}-L_{y}^{-1} L_{z}^{-1} \int_{\xi^{n}-\frac{3}{8} L_{x}, 0,0}^{\xi^{n}+\frac{3}{8} L_{x}, L_{y}, L_{z}} c\left(\mathbf{x}, t^{n+1}\right) d \mathbf{x}
$$

because a region of $x \in\left[\xi-\frac{3}{8} L_{x}, \xi+\frac{3}{8} L_{x}\right]$ was wide enough to cover entire front brush in each studied case. The remaining two regions $x \in\left(\xi-\frac{1}{2} L_{x}, \xi-\frac{3}{8} L_{x}\right)$ and $x \in\left(\xi+\frac{3}{8} L_{x}, \xi+\frac{1}{2} L_{x}\right]$ represent the pure unburned and burned states, respectively, and do not affect the motion of the front $G(\mathbf{x}, t)=0$. The statistics is sampled by averaging over $y$ - $z$ planes and using a new $x$-coordinate mapping $\left(\xi-\frac{1}{2} L_{x}, \xi+\frac{1}{2} L_{x}\right)$ to $\left(0, L_{x}\right)$.

Eight 3D-DNS cases were simulated by varying $U_{0}^{\prime}{ }_{0} / S_{L}=1,2,5$, and 10 with $\operatorname{Re}=U_{0}^{\prime} \lambda_{0} / v$ $=100$ or 50 . The Reynolds number was changed by changing the scale $L$ of the computational domain. In all cases, $U_{0}^{\prime}$ was the same and it will be used to normalize velocity in the following. In all cases, the time step was set as $\Delta t=0.029 \cdot \Delta x / U_{0}^{\prime}$ where $\Delta x$ is the cell size. For constant-density system, the simulated turbulence fields were statistically the same for various $S_{L}$ at same Re. The forced turbulence developed into statistically stationary state with a ratio of an adapted turbulent integral scale $\lambda$ to $L$ being 0.228 and 0.256 for $\mathrm{Re}=100$ and 50, respectively. The obtained ratio is within previously reported ranges of $0.17-0.28$, cf. Table 2 , column $k_{0} l$ in Ref. 31 . The Reynolds number based on the adapted integral scale was $\operatorname{Re}_{\lambda}=U_{0}^{\prime} \lambda / \nu=91$ and 51, respectively. The ratio of $\lambda$ to the Kolmogorov scale $\eta=\left(v^{3} /\langle\varepsilon\rangle\right)^{1 / 4}$ was 33.8 and 24.4 for $\operatorname{Re}=100$ and 50, respectively, and $\Delta x$ was of the order of $\eta$. Starting from the initial field each simulation was run 5 initial eddy turnover times $\tau_{0}=\lambda_{0} / U_{0}^{\prime}$ in order for the forced turbulence to develop. For sampling converged statistics, each simulation was run at least $100 \tau_{0}$.

\section{RESULTS AND DISCUSSIONS}

Figure 1 shows typical instantaneous 3D front and turbulence field $\left(\lambda_{2}\right.$-vortexes $\left.{ }^{32}\right)$. Comparison of left and right images shows that a decrease in $S_{L}$ results in more broken and wrinkled front surface and thicker front brush. Homogeneous distribution of tanged vortexes indicates that the simulated turbulence is "physical" and homogenous forcing is achieved. The homogeneity $\left(\overline{u^{2}}=\overline{w^{2}}=1\right)$ and
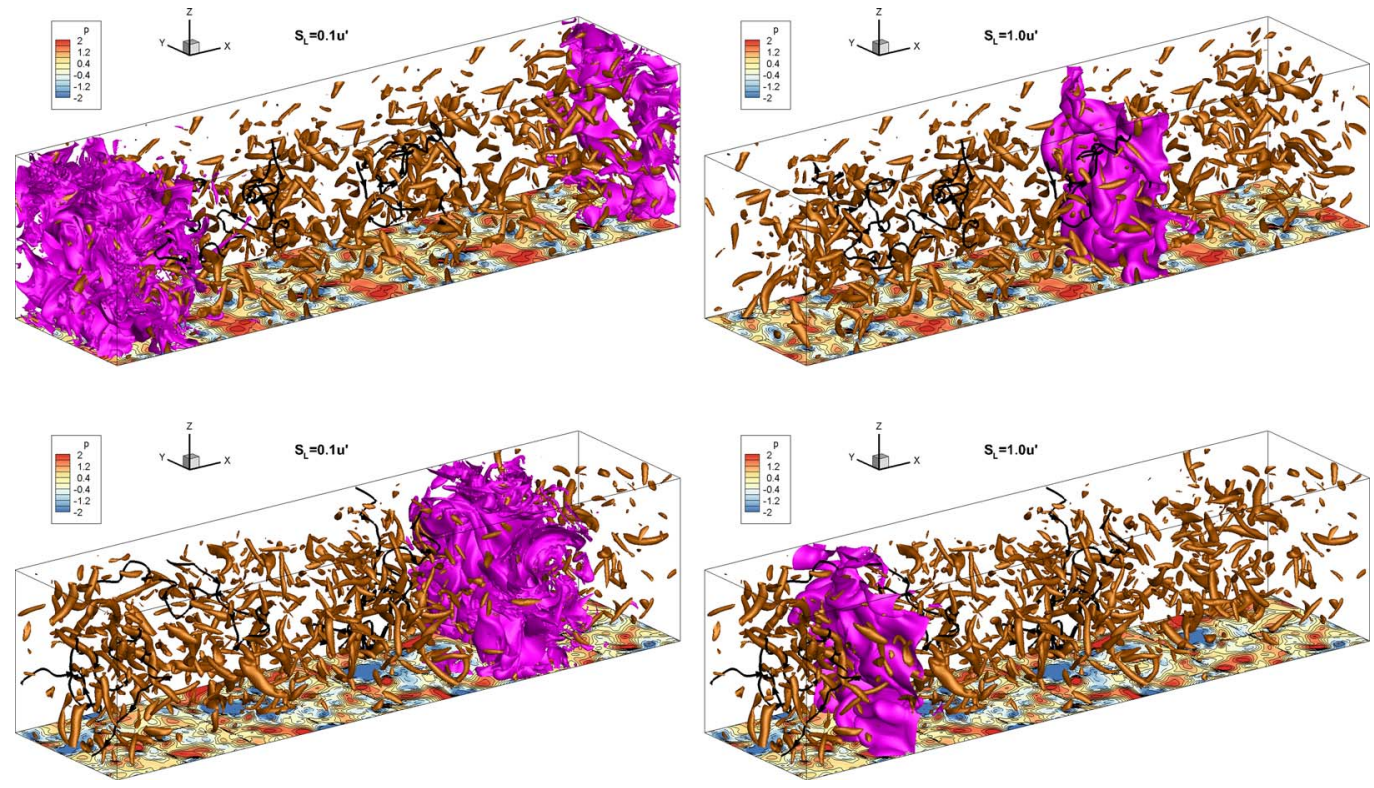

FIG. 1. Instantaneous 3D front $\left(G=0\right.$ isosurface, purple) and turbulence field ( $\lambda_{2}$-vortex tubes) for $U_{0}^{\prime} / S_{L}=10$ (left) and $U_{0}^{\prime} / S_{L}=1$ (right) at $\mathrm{Re}=100$. Top and bottom rows are from two different time instants. The solid (black) line is instantaneous stream line. The floor plane shows the fluctuating pressure contour. 


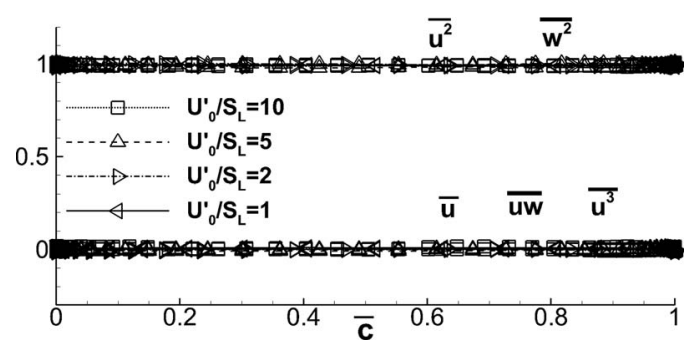

FIG. 2. Unconditioned flow statistics $\left(\bar{u}, \overline{u^{2}}, \overline{w^{2}}, \overline{u w}, \overline{u^{3}}\right)$ vs. $\bar{c}$ for various $S_{L}$ at $\operatorname{Re}=100$.

isotropy $\left(\overline{u w}=0\right.$ and $\left.\overline{u^{3}}=0\right)$ of the simulated turbulence is confirmed in Fig. 2. Here, $u$, $v$, and $w$ designate components of velocity vector in $x$-, $y$-, and $z$-directions, respectively, with mean flow velocity vanishing.

\section{A. Unconditioned statistics}

Figure 3 shows that the normalized mean front brush thickness $\delta_{t}=(\max (\partial \bar{c} / \partial x) \lambda)^{-1}$ is significantly increased when $U_{0}^{\prime} / S_{L}$ is increased, contrary to wide-spread belief that mean flame brush thickness is mainly controlled by the turbulent integral scale $\lambda$ (note that $\lambda$ is not affected by $U_{0}^{\prime} / S_{L}$ in the present DNS, but depends on Re). Although such a trend could be expected, because the thickness should grow to infinity as $S_{L} \rightarrow 0$, only two models of premixed turbulent combustion yield expressions that are qualitatively consistent with data plotted in Fig. 3 (note that these two models do not address the influence of heat release on the turbulent flow, similarly to the present DNS). In particular, increasing dependencies of (i) $\delta_{t} \propto U_{0}^{\prime} / S_{L}$ or (ii) $\delta_{t} \propto\left(U_{0}^{\prime} / S_{L}\right)^{1 / 2}$ have earlier been predicted by hypothesizing either (i) that the rate of the growth of turbulent flame brush due to turbulent diffusion is equal to $S_{L}$ in a fully-developed flame ${ }^{33}$ or (ii) that flame surface produced by large-scale turbulent eddies is periodically consumed due to self-propagation of flamelets, ${ }^{34}$ respectively. The present DNS data are best fitted (curve) using a weaker scaling $\delta_{t} \propto\left(U_{0}^{\prime} / S_{L}\right)^{q}$ with $q \approx$ $1 / 3$. The present authors are not aware of an expression or a model that yields or explains this scaling. Therefore, Fig. 3 appears to challenge turbulent combustion community and requires explanation.

Figure 4 shows that the direction of turbulent flux $\overline{u^{\prime} c^{\prime}}$ is predicted by the gradient diffusion model, i.e., $\overline{u^{\prime} c^{\prime}}=-D_{t} \partial \bar{c} / \partial x$. However, contrary to inert turbulent flows, the coefficient $D_{t}$ in this closure relation depends not only on turbulence characteristics, but also is weakly increased by $\bar{c}$ and is significantly decreased when $S_{L}$ is increased.

If we associate front self-propagation simulated by us with chemical reactions that are localized to the front, but not resolved within the framework of the level-set approach, then, DNS data plotted in Fig. 4 are consistent with contemporary knowledge on the influence of chemical reactions on

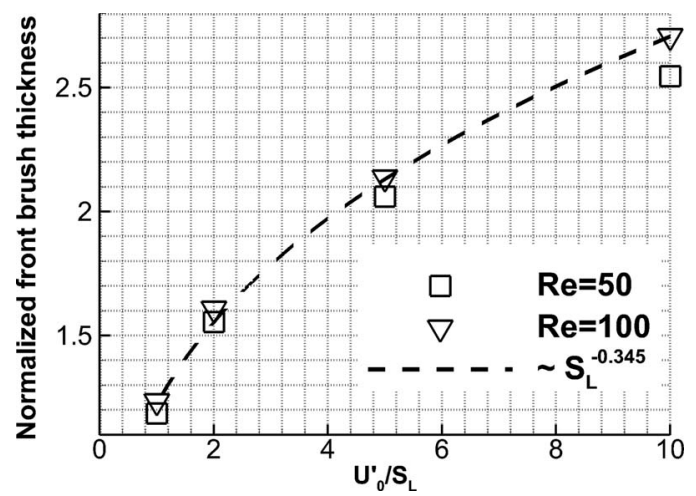

FIG. 3. Normalized mean front brush thickness vs. $U^{\prime}{ }_{0} / S_{L}$. Curve approximates DNS data shown in symbols. 


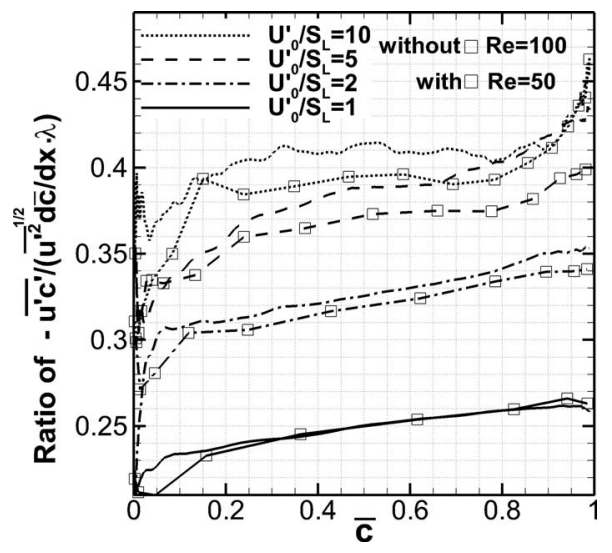

FIG. 4. Ratio of $-\overline{u^{\prime c l}} /\left(\overline{u^{\prime 2}} \frac{1}{2} \cdot \partial \bar{c} / \partial x \cdot \lambda\right)$ vs. $\bar{c}$.

turbulent mixing in constant-density flows. Such an influence was known after the pioneering work by Corrsin ${ }^{35}$ and was addressed in the combustion literature long time ago. ${ }^{36}$ In particular, balance equations for conditioned velocities indicate that source and sink terms conditioned to flamelets reduce the magnitude of turbulent scalar flux even in the constant-density case, ${ }^{37,38}$ with these terms being proportional to the mean rate of product creation $\bar{W}$. Within the framework of the paradigm of infinitely thin flame front, $\bar{W}=\rho_{u} S_{L} \Sigma_{f}$ and the reduction effect is increased by the front speed, in line with the present DNS data. Here, $\Sigma_{f}$ is the flame-front surface density.

\section{B. Conditioned statistics}

Figure 5 shows profiles of conditioned first moments obtained in the present 3D (curves) and previous 2D (squares and circles) DNS. ${ }^{12}$ Moreover, results of a simple simulation ${ }^{6}$ of a laminar flame stabilized in a periodical shear 1D flow are reported (triangles). In line with the gradient scalar flux and constraint of $0=\bar{u}=(1-\bar{c}) \bar{u}_{u}+\bar{c} \cdot \bar{u}_{b}$, the conditioned velocity $\bar{u}_{u}$ is positive, but $\bar{u}_{b}$ stays negative. The previous $2 \mathrm{D}$ results are rather consistent with current $3 \mathrm{D}$ ones when $U_{0}^{\prime} / S_{L}$ $=1$, but underpredict the difference $\bar{u}_{u}-\bar{u}_{b}$ when $U_{0}^{\prime} / S_{L}=10$. To the contrary, the simple laminar model overpredicts the difference, with the effect being more pronounced at smaller $S_{L}$.
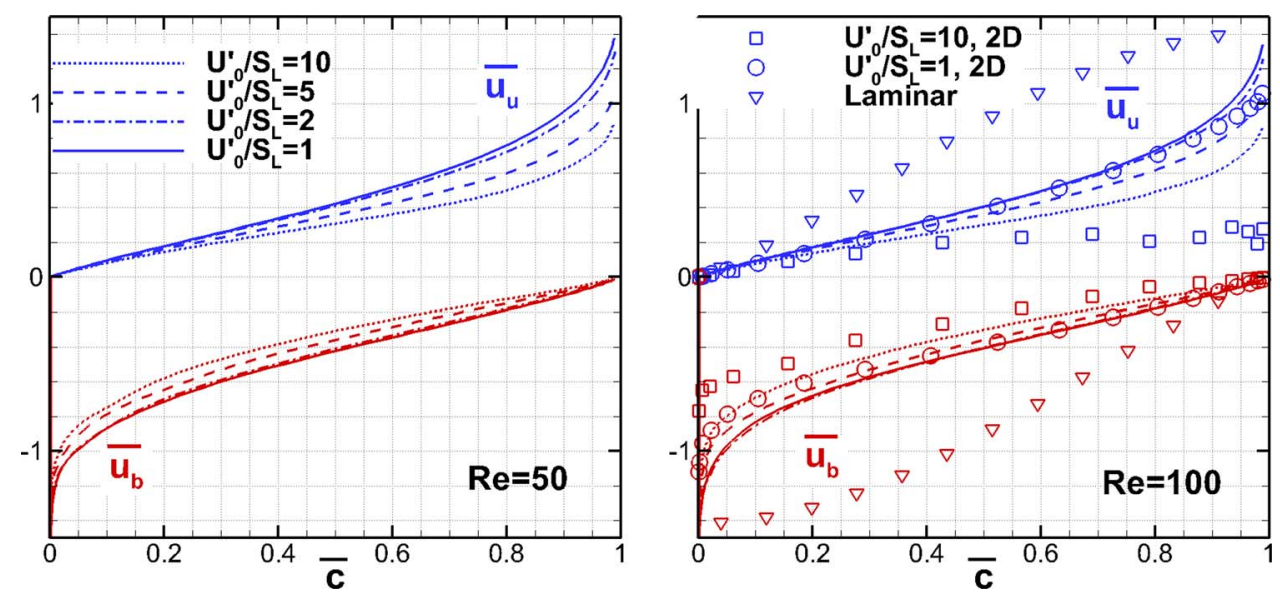

FIG. 5. Dotted, dashed, dotted-dashed, and solid curves show conditioned first moments $\bar{u}_{u} \geq 0$ (blue) and $\bar{u}_{b} \leq 0$ (red) obtained in the present 3D-DNS for $U_{0}^{\prime} / S_{L}=10,5,2$, and 1, respectively, and Re $=50$ (left) or 100 (right). Squares and circles show $2 \mathrm{D}$ DNS results ${ }^{12}$ with $U_{0}^{\prime}{ }_{0} / S_{L}=10$ and 1 , respectively. The latter results are normalized using the local $\left(\overline{u^{\prime 2}}\right)^{\frac{1}{2}}$, which decays with $\bar{c}$ in the 2D case. Triangles refer to a simple laminar flame studied in Ref. 6. 

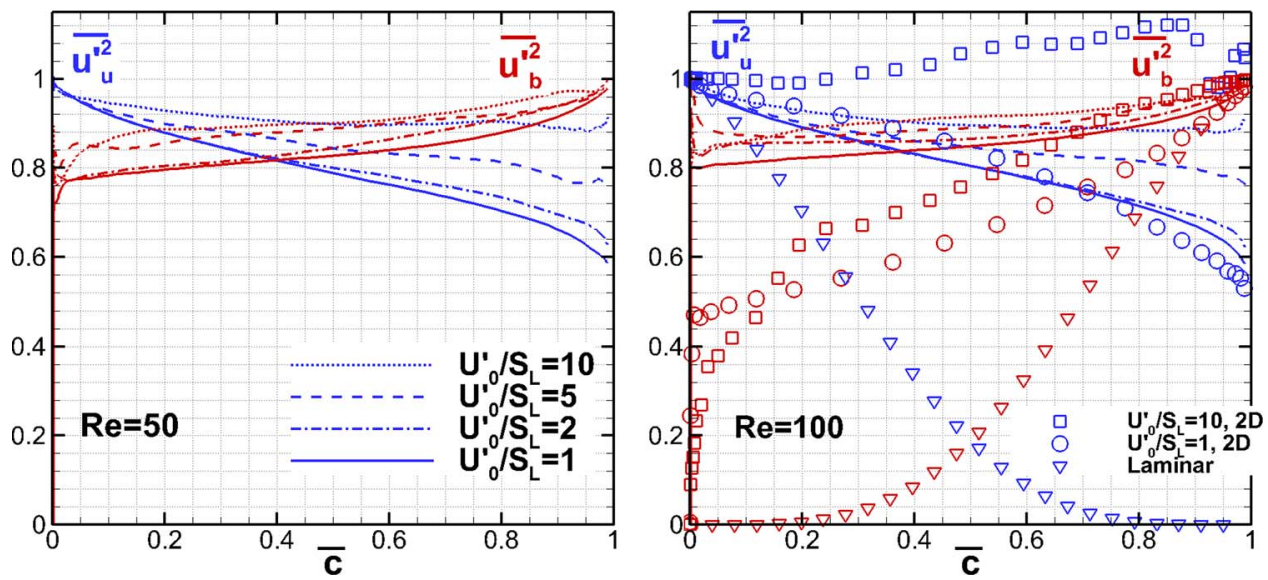

FIG. 6. Conditioned second moments, $\left(\overline{u^{\prime 2}}\right)_{b}$ and $\left(\overline{u^{\prime 2}}\right)_{u}$. Legends are explained in caption to Fig. 5.

Figure 6 shows that $\left(\overline{u^{\prime 2}}\right)_{b}$ and $\left(\overline{u^{\prime 2}}\right)_{u}$ decrease when $\bar{c}$ increases and decreases, respectively. These normalized conditioned second moments differ from the true turbulent second moment (unity). The difference depends weakly on Re, is increased by $S_{L}$, is more pronounced for $\left(\overline{u^{\prime 2}}\right)_{u}$ when compared to $\left(\overline{u^{\prime 2}}\right)_{b}$ and in the $2 \mathrm{D}$ case when compared to the $3 \mathrm{D}$ case.

Figure 7 shows that, contrary to the true turbulent third moment $\overline{u^{\prime 3}}=0$, the conditioned moments do not vanish, with $\left(\overline{u^{\prime 3}}\right)_{u}$ and $\left(\overline{u^{\prime 3}}\right)_{b}$ being negative and positive, respectively, in the largest part of the mean front brush. At $U_{0}^{\prime}{ }_{0} / S_{L}=10$, the effect is more pronounced for $\left(\overline{u^{\prime 3}}\right)_{u}$ than for $\left(\overline{u^{\prime 3}}\right)_{b}$, but $\left|\left(\overline{u^{\prime 3}}\right)_{u}\right|$ is clearly decreased when $S_{L}$ is decreased, whereas the influence of $S_{L}$ on $\left|\left(\overline{u^{\prime 3}}\right)_{b}\right|$ is substantially weaker. The absolute values of the conditioned third moments are increased by Re. Moreover, $\left|\left(\overline{u^{\prime 3}}\right)_{u}\right|$ and $\left|\left(\overline{u^{\prime 3}}\right)_{b}\right|$ decrease when $\bar{c}$ decreases and increases, respectively. One of the present authors ${ }^{38}$ argued recently that conditioned third moments should not vanish in isotropic constant-density turbulence, and this prediction is confirmed by the present DNS.

Previous model studies ${ }^{6,11}$ and the present 3D DNS clearly show that conditioned moments of velocity field are not true turbulence characteristics within the mean front brush. To the contrary, profiles of the mean and conditioned enstrophy $\frac{1}{2} \overline{\omega^{2}}=\frac{1}{2} \overline{(\nabla \times \mathbf{u})^{2}}$ reported in Fig. 8(a) indicate that these quantities are sufficiently close to each other in all investigated cases, while differences in $\overline{\omega^{2}}$ and $\left(\overline{\omega^{2}}\right)_{u}$ are pronounced in a wider range of $\bar{c}$ when compared to differences in $\overline{\omega^{2}}$ and $\left(\overline{\omega^{2}}\right)_{b}$. In all studied cases, $\overline{\omega^{2}} \approx\left(\overline{\omega^{2}}\right)_{u}$ if $\bar{c}<0.5$ and $\overline{\omega^{2}} \approx\left(\overline{\omega^{2}}\right)_{b}$ if $\bar{c}>0.5$. Therefore, such a
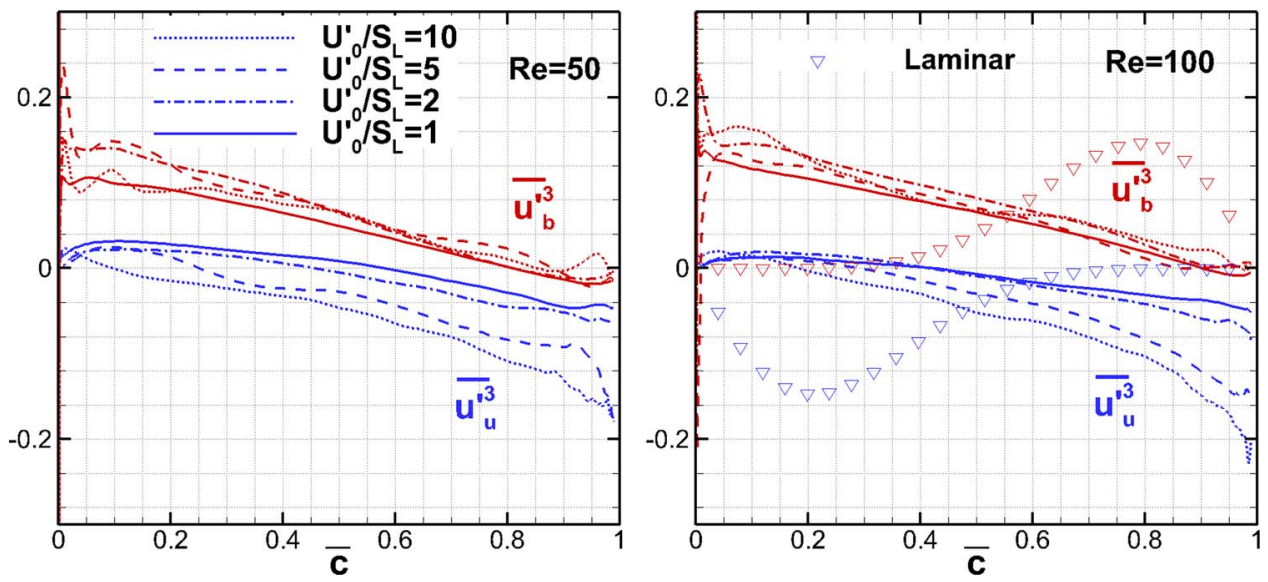

FIG. 7. Conditioned third moments, $\left(\overline{u^{\prime 3}}\right)_{b}$ and $\left(\overline{u^{\prime 3}}\right)_{u}$. Legends are explained in caption to Fig. 5 . 

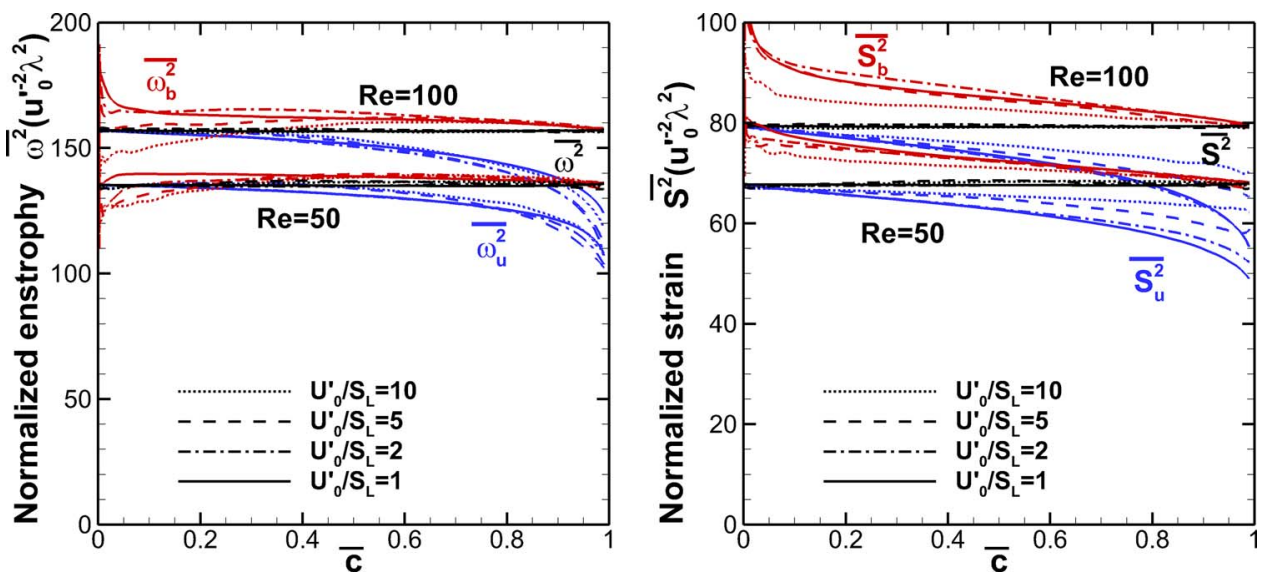

FIG. 8. Normalized unconditioned/conditioned enstrophy (left) $\overline{\omega^{2}}$ (black), $\left(\overline{\omega^{2}}\right)_{b}\left(\right.$ red), and $\left(\overline{\omega^{2}}\right)_{u}$ (blue) and total strain (right) $\overline{S_{i j}^{2}}$ (black), $\left(\overline{S_{i j}^{2}}\right)_{b}$ (red), and $\left(\overline{S_{i j}^{2}}\right)_{u}$ (blue).

combination of conditioned enstrophies appears to be a proper choice for characterizing the local true turbulence within a premixed front brush at least in a constant-density case. Figure 8(b) shows that the difference between unconditioned and conditioned total strain (or dissipation) rates $\overline{S_{i j}^{2}}=$ $\bar{\varepsilon} / 2 v=\overline{\left(\frac{1}{2}\left(\nabla+\nabla^{T}\right) \mathbf{u}\right)^{2}}$ is larger when compared to enstrophy.

\section{CONCLUSIONS}

A numerical tool has been developed for fully periodical 3D DNS study of a statistically planar, one-dimensional front that self-propagates in statistically stationary, homogeneous, isotropic, forced turbulence in the constant-density case.

Obtained numerical results show the following trends:

- Mean front brush thickness $\boldsymbol{\delta}_{t}$ is significantly increased when a ratio of the rms turbulent velocity to the front speed is increased from 1 to 10 . The obtained trend does not support widely accepted assumption that the mean flame brush thickness is controlled by turbulence integral length scale. Although the gradient diffusion closure yields the right direction of turbulent scalar flux, the diffusion coefficient $D_{t}$ depends not only on turbulence characteristics, but also on the mean progress variable. Moreover, $D_{t}$ is decreased when the front speed is increased. Thus, front propagation affects turbulent scalar transport even in a constant-density case.

- Conditioned moments of the velocity field differ from counterpart mean moments, with the latter moments characterizing the true turbulence in the studied cases. This result validates earlier model studies that disputed the use of conditioned velocity moments for characterizing turbulence within a premixed flame brush.

- Computed conditioned enstrophies are close to the mean enstrophy in all studied cases, thus, suggesting the use of conditioned enstrophy for characterizing turbulence within a premixed flame brush, at least in a hypothetical constant-density case.

Variable-density effects will be addressed in a subsequent study.

\section{ACKNOWLEDGMENTS}

The first and third authors were sponsored by the Swedish Research Council (VR) and the national Centre for Combustion Science and Technology (CeCOST). The second author (A.L.) was supported by the Swedish Energy Agency and by the Chalmers Combustion Engine Research Center (CERC). The computation was performed using the computer facilities provided by the Centre for Scientific and Technical Computing at Lund University (LUNARC). 
${ }^{1}$ A. N. Lipatnikov and J. Chomiak, "Turbulent flame speed and thickness: Phenomenology, evaluation, and application in multi-dimensional simulations," Prog. Energy Combust. Sci. 28, 1 (2002).

${ }^{2}$ D. Veynante and L. Vervisch, "Turbulent combustion modeling," Prog. Energy Combust. Sci. 28, 193 (2002).

${ }^{3}$ J. Janicka and A. Sadiki, "Large eddy simulation of turbulent combustion systems," Proc. Combust. Inst. 30, 537 (2005).

${ }^{4}$ H. Pitsch, "Large eddy simulation of turbulent combustion," Annu. Rev. Fluid Mech. 38, 453 (2006).

${ }^{5}$ A. N. Lipatnikov and J. Chomiak, "Effects of premixed flames on turbulence and turbulent scalar transport," Prog. Energy Combust. Sci. 36, 1 (2010).

${ }^{6}$ A. N. Lipatnikov, "Can we characterize turbulence in premixed flames?" Combust. Flame 156, 1242 (2009).

${ }^{7}$ K. N. C. Bray, P. A. Libby, and J. B. Moss, "Unified modeling approach for premixed turbulent combustion-Part I: General formulation," Combust. Flame 61, 87 (1985).

${ }^{8}$ P. A. Libby, "On the prediction of intermittent turbulent flows," J. Fluid Mech. 68, 273 (1975).

${ }^{9}$ C. Dopazo, "On conditioned averages for intermittent turbulent flows," J. Fluid Mech. 81, 433 (1977).

${ }^{10}$ A. N. Lipatnikov, "Conditionally averaged balance equations for modeling premixed turbulent combustion in flamelet regime," Combust. Flame 152, 529 (2008).

${ }^{11}$ A. N. Lipatnikov, "Conditioned moments in premixed turbulent reacting flows," Proc. Combust. Inst. 33, 1489 (2011).

${ }^{12}$ R. Yu, A. N. Lipatnikov, and X. S. Bai, "Conditioned moments in turbulent premixed flames: A DNS study," in Proceedings of the Eighth Mediterranean Combustion Symposium, 8-13 September 2013, edited by N. Selçuk, F. Beretta, M. S. Mansour, and A. D'Anna (Çeşme, Izmir, Turkey, 2013), paper TC-2, see also the conference website is: http://www.ichmt.org/mcs-13/.

${ }^{13}$ G. Boffetta and R. E. Ecke, "Two-dimensional turbulence," Annu. Rev. Fluid Mech. 44, 427 (2012).

${ }^{14}$ J. B. Bell, M. S. Day, J. F. Grcar, and M. J. Lijewski, "Active control for statistically stationary turbulent premixed flame simulations," Commun. Appl. Math. Comput. Sci. 1, 29 (2006).

${ }^{15}$ E. D. Siggia, "Numerical study of small-scale intermittency in three-dimensional turbulence," J. Fluid Mech. 107, 375 (1981).

${ }^{16}$ F. A. Williams, Combustion Theory, 2nd ed. (Benjamin/Cummings, California, 1985).

${ }^{17}$ P. Clavin, "Dynamical behavior of premixed flame fronts in laminar and turbulent flows," Prog. Energy Combust. Sci. 11, 1 (1985).

${ }^{18}$ R. Yu, J. Yu, and X. S. Bai, "An improved high-order scheme for DNS of low Mach number turbulent reacting flows based on stiff chemistry solver," J. Comput. Phys. 231, 5504 (2012).

${ }^{19}$ R. Yu and X. S. Bai, "Direct numerical simulation of lean hydrogen/air auto-ignition in a constant volume enclosure," Combust. Flame 160, 1706 (2013).

${ }^{20}$ J. Yu, R. Yu, X. Q. Fan, M. Christensen, A. A. Konnov, and X. S. Bai, "Onset of cellular flame instability in adiabatic $\mathrm{CH} 4 / \mathrm{O} 2 / \mathrm{CO} 2$ and $\mathrm{CH} 4 /$ air laminar premixed flames stabilized on a flat-flame burner," Combust. Flame 160, 1276 (2013).

${ }^{21}$ F. Zhang, R. Yu, and X. S. Bai, "Detailed numerical simulation of syngas combustion under partially premixed combustion engine conditions," Int. J. Hydrogen Energy 37, 17285 (2012).

${ }^{22} \mathrm{H}$. Carlsson, R. Yu, and X. S. Bai, "Direct numerical simulation of lean premixed CH4/air and H2/air flames at high Karlovitz numbers" (submitted).

${ }^{23}$ R. Yu and X. S. Bai, “A semi-implicit scheme for large eddy simulation of piston engine flow and combustion,” Int. J. Numer. Meth. Fluids 71, 13 (2013).

${ }^{24}$ R. Yu and X. S. Bai, "A fully divergence-free method for generation of inhomogeneous and anisotropic turbulence with large spatial variation,” J. Comput. Phys. 256, 234 (2014).

${ }^{25}$ S. Ghosal, T. S. Lund, P. Moin, and K. Akselvoll, "A dynamic localization model for large-eddy simulation of turbulent flows," J. Fluid Mech. 286, 229 (1995).

${ }^{26}$ P. Wang, "Large eddy simulation of turbulent swirling flows and turbulent premixed combustion," Ph.D. thesis (Lund University, 2005).

${ }^{27}$ G. S. Jiang and D. Peng, "Weighted ENO schemes for Hamilton-Jacobi equations," SIAM J. Sci. Comput. 21, 2126 (2000).

${ }^{28}$ S. Gottlieb and C. W. Shu, "Total variation diminishing Runge-Kutta schemes," Math. Comput. 67, 73 (1998).

${ }^{29}$ G. Russo and P. Smereka, "A remark on computing distance functions," J. Comput. Phys. 163, 51 (2000).

${ }^{30}$ M. B. Sun, Z. G. Wang, and X. S. Bai, "Assessment and modification of sub-cell-fix method for re-initialization of level-set distance function," Int. J. Numer. Meth. Fluids 62, 211 (2010).

${ }^{31}$ V. Eswaran and S. B. Pope, "An examination of forcing in direct numerical simulations of turbulence," Comput. Fluids 16, 257 (1988).

${ }^{32}$ J. Jeong and F. Hussain, "On the identification of a vortex," J. Fluid Mech. 285, 69 (1995).

${ }^{33}$ V. L. Zimont, "Gas premixed combustion at high turbulence. Turbulent flame closure combustion model," Exp. Therm. Fluid Sci. 21, 179 (2000).

${ }^{34}$ A. M. Klimov, "Flame propagation in intense turbulence," Dokl. Akad. Nauk SSSR 221/1, 56 (1975).

${ }^{35}$ S. Corrsin, "Limitations of gradient transport models in random walks and in turbulence," Adv. Geophys. A 18, 25 (1975).

${ }^{36}$ R. Borghi and D. Dutoya, "On the scales of the fluctuations in turbulent combustion," Proc. Combust. Inst. 17, 235 (1978).

${ }^{37}$ A. N. Lipatnikov, "Transient behavior of turbulent scalar transport in premixed flames," Flow Turbul. Combust. 86, 609 (2011).

${ }^{38}$ A. N. Lipatnikov, Fundamentals of Premixed Turbulent Combustion (CRC Press, Florida, 2012). 